\title{
Clinical profile of children with renal disease admitted to a tertiary care centre in Sri Lanka
}

\author{
*Manoji Gitanjali Sathiadas ${ }^{1}$, Archchana Anantharajah ${ }^{1}$, Niroshan $\operatorname{Vadivel}^{1}$
}

Sri Lanka Journal of Child Health, 2021; 50(1): 87-93

\begin{abstract}
Introduction: In Sri Lanka, renal diseases are increasing in the adult population. Spectrum of renal disease is different in children when compared to adults.
\end{abstract}

Objectives: To determine the disease burden of paediatric renal diseases, spectrum and clinical presentation.

Method: A retrospective study was done using the electronic database maintained in the paediatric professorial unit, Teaching Hospital Jaffna. Data of patients admitted from January 2015 to December 2018 were analysed using the software available.

Results: Renal diseases accounted to 517 (3.9\%) cases of total admissions during the study period. The male: female ratio was $1: 1.1$. Mean age of the patients was $3.8 \pm 1.7$ years. Urinary tract infection (UTI) was the most common disease $(55.9 \%)$ followed by nephrotic syndrome $(17.6 \%)$ and acute glomerulonephritis $(13.9 \%)$. Urine culture was positive in $40 \%$ of the patients treated for UTI and imaging studies were done in $90 \%$. Majority $(94.6 \%)$ of the nephrotic syndrome were steroid responsive and renal biopsy was performed in $23 \%$. Chronic kidney disease $(3.9 \%)$ and renal tract anomalies (1.9\%) contributed to the disease load as well. The overall outcome of the renal diseases indicated that majority $(49.9 \%)$ were on regular follow-up and $6.1 \%$ were referred to specialised renal units. Four $(0.7 \%)$ died due to renal diseases.

Conclusions: Renal diseases accounted for 3.9\% of total admissions during the study period. UTI was the commonest disease $(55.9 \%)$ followed by nephrotic syndrome $(17.6 \%)$ and acute glomerulonephritis (13.9\%).

${ }^{1}$ University of Jaffna, Sri Lanka

*Correspondence: docsathiadas@hotmail.com

D https://orcid.org/0000-0002-7822-0447 (Keceived on 19 March 2020: Accepted after revision on 22 May 2020)

The authors declare that there are no conflicts of interest

Personal funding was used for the project.

Open Access Article published under the Creative

Commons Attribution CC-BY CC) (i) License
DOI: http://dx.doi.org/10.4038/sljch.v50i1.9408

(Key words: Renal diseases, paediatrics, Jaffna, Sri Lanka)

\section{Introduction}

The spectrum of diseases involving the renal tract varies in respect of the geographical location, environmental factors and genetic predisposition. Worldwide, the common reason for hospitalization of children with renal involvement is infections of the urinary $\operatorname{tract}^{1}$. It has been reported in the literature that renal diseases are on the rise in this part of world and is a major cause of morbidity and mortality ${ }^{1}$. Chronic kidney disease of unknown aetiology $(\mathrm{CKDu})$ has increased the morbidity and mortality of this country and an aetiology related to ground water has been extensively studied especially in the adult population ${ }^{2}$. In some of the studies it is postulated that the insult may have occurred in childhood and progress to chronic kidney disease in adulthood. This is especially noted in children living in $\mathrm{CKDu}$ endemic regions of the country ${ }^{3}$. Identifying these insults early may help to delay the progression of kidney damage ${ }^{4,5}$.

Paediatric patients with renal diseases, especially very young children, may present with nonspecific signs and symptoms unrelated to the urinary tract. Unexplained fever or failure to thrive may be the only manifestation ${ }^{4}$. Paediatricians, therefore, should be familiar with the modes of presentation of renal disease and should have a high index of suspicion of these conditions ${ }^{4}$. Studies from different geographical areas around the world have reported variable prevalence patterns of renal diseases. In most areas urinary tract infections (UTIs) are common whereas in some areas acute glomerular nephritis, nephrotic syndrome and chronic kidney disease have been reported as common. Theses variations could be related to different genetic predisposition, environmental factors or lack of awareness about importance of early diagnosis of such disorders ${ }^{1,4}$.

A literature search using PubMed and EMBASE showed that the spectrum of renal diseases in hospitalised children in Sri Lanka was none but searches mentioning specific diseases like nephrotic syndrome and urinary tract infection (UTI). Children with confirmed UTI have shown that the common presenting feature was fever and 
the common organism was coliforms ${ }^{6}$. Searches on nephrotic syndrome showed several studies related to the management of it and practical variations from the International Study of Kidney Disease in Children (ISKDC) regime ${ }^{7}$. Knowledge about the pattern of the diseases is important to develop facilities like providing training to healthcare providers, developing laboratory services that aid in diagnosis and designing infrastructure. A treating physician should have high index of suspicion and the knowledge of various presentations of the renal diseases in children ${ }^{3}$.

\section{Objectives}

The objectives of the study were to identify the spectrum of renal diseases in children admitted to hospital, the socio-demographic profile, factors associated with renal diseases like family history, presenting clinical features of each renal diseases, investigations performed and to assess the overall outcome of these patients.

\section{Method}

A retrospective descriptive cross-sectional study was done at the Professorial Paediatric unit, Teaching Hospital Jaffna. Data were extracted from the electronic database maintained at the unit over a four-year period from January 2015 to December 2018. The electronic patient management system, which was developed and implemented in 2014 onwards, uses a report generator. Data were then exported from the electronic report to Microsoft Excel 2016 using a built-in interphase in the system. Children admitted with renal diseases were filtered and data regarding clinical history, sociodemographic profile, examination findings, investigations, including imaging, renal biopsy, treatment options and disease outcome, were considered. Readmissions were excluded into the total count of the patients. Any renal diseases ending in renal failure were considered as chronic renal failure. The diagnosis entered into the database was considered to classify the patient's diseases. This diagnosis was based on history, physical examination and laboratory investigations. This was also matched to the ICD 10 classifications of the renal diseases. Chronic kidney disease (CKD) was defined based on Kidney Disease Outcomes Qualitative Initiative (KDOQI) ${ }^{8,9}$ and nephrotic syndrome was defined based the International Study of Kidney Disease in Children (ISKDC) guidelines ${ }^{10}$.

Maternal education and household income were used as indicators of the socio-economic status of the family. Maternal education was categorised as no education, primary-incomplete, primarycompete, secondary-incomplete, secondarycomplete and higher degree. For household income, participants were asked to select their monthly average income in Sri Lankan Rupees (USD $1=$ Rupees 150) from the following categories: $<15,000,15,000-50,000$ and $>50,000$. The water source for drinking was categorized into ground, tap and mineral water.

Ethical issues: Ethical clearance was obtained from the Ethical Review Committee of the Faculty of Medicine, University of Jaffna. (J/ERC/19/ $105 / \mathrm{NDR} / 0213)$. As it was a retrospective study, informed consent was not possible. Confidentiality is assured.

Statistical analysis: This was done using the Statistical Package for Social Studies (SPSS 22) Frequencies and percentages were used in the analysis. The disease burden of paediatric renal disease was calculated as the proportion of the total number of paediatric admissions to the number diagnosed with a renal condition.

\section{Results}

A total of 13,311 admissions were considered and 544 data were filtered using the software package. A total of $517(95 \%)$ with renal diseases were considered and the rest $(n=27)$ were not considered due to incomplete data. A total of 6 hours was spent to retrieve and categorise the data from the patient management system. Renal disease burden was $3.9 \%$ for the whole population and the annual renal disease burden ranged from $3.7 \%$ to $4.4 \%$ (Table 1).

Table 1: Paediatric renal disease admissions during the study period

\begin{tabular}{|l|c|c|c|}
\hline Year & $\begin{array}{c}\text { Total number of } \\
\text { admitted patients }\end{array}$ & $\begin{array}{c}\text { Total number of new patients } \\
\text { admitted with renal diseases }\end{array}$ & $\begin{array}{c}\text { Percentage of patients with } \\
\text { renal diseases }\end{array}$ \\
\hline 2015 & 2783 & 123 & 4.42 \\
\hline 2016 & 2917 & 111 & 3.81 \\
\hline 2017 & 3975 & 149 & 3.75 \\
\hline 2018 & 3636 & 134 & 3.69 \\
\hline Total & 13,311 & 517 & 3.88 \\
\hline
\end{tabular}

The mean age of the population was $3.8 \pm 1.7$ years. There were $270(52.3 \%)$ boys and $247(47.7 \%)$ girls with a male: female ratio of $1: 1.1$. The family type was extended in $51.6 \%$ of the patients and
$52 \%$ of the mothers had not completed secondary education. Majority (63.8\%) obtained their drinking water from an underground source. (Table 2) 
Table 2: Socio-demographic features of the patients

\begin{tabular}{|l|c|l|c|}
\hline \multicolumn{1}{|c|}{ Socio-demographic feature } & Number (\%) & \multicolumn{1}{|c|}{ Socio-demographic feature } & Number (\%) \\
\hline Age range (years) & $150(29.0)$ & Gater source for drinking purposes & \\
Less than 1 & $247(47.8)$ & Tap water - from common source & $330(63.8)$ \\
$1-5$ & $120(23.2)$ & Mineral water & $85(16.4)$ \\
More than 5 & $32(06.2)$ & Household income (Rupees) & $102(19.7)$ \\
\hline Family history of renal disease & $485(93.8)$ & $15,000-50,000$ & $204(39.5)$ \\
Positive & & More than 50,000 & $223(43.1)$ \\
Negative & $267(51.6)$ & Maternal educational level & $90(17.4)$ \\
& $250(48.4)$ & Primary incomplete & $24(04.6)$ \\
Family type & Primary complete & $57(11.0)$ \\
Nuclear & Secondary incomplete & $188(36.4)$ \\
Extended & Secondary complete & $203(39.3)$ \\
& & Higher degree & $45(08.7)$ \\
\hline
\end{tabular}

Our analysis shows that UTI was the major renal disease comprising $56 \%$ of all admissions followed by nephrotic syndrome comprising $17.6 \%$ and acute glomerular nephritis, 14\%. The disease spectrum is shown in table 3 .

Table 3: Spectrum of paediatric kidney diseases

\begin{tabular}{|l|c|}
\hline \multicolumn{1}{|c|}{ Renal disease (ICD 10 Number) } & Number (\%) \\
\hline Urinary tract infection (N39) & $289(55.9)$ \\
\hline Nephrotic syndrome (N04.9) & $91(17.6)$ \\
\hline Acute glomerular nephritis (N00.9) & $72(13.9)$ \\
\hline Chronic renal failure (N18.9) & $20(03.9)$ \\
\hline Acute renal failure (N17.9) & $06(01.2)$ \\
\hline Congenital anomalies of the renal tract(Q64.9) & $10(01.9)$ \\
\hline Tubular disorders including renal tubular acidosis (N25.89) & $09(01.9)$ \\
\hline Hemolytic uremic syndrome (D59.3) & $01(0.2)$ \\
\hline Malignancy of the renal tract (C67.9) & $04(0.8)$ \\
\hline Renal Calculi (N20.0) & $07(01.3)$ \\
\hline Hypercalciuria (R82.994) & $04(0.8)$ \\
\hline Isolated Haematuria (R31.9) & $04(0.8)$ \\
\hline
\end{tabular}

Family history of renal disease was seen in 32 $(6.2 \%)$ patients and the presence of renal calculi in the first degree relative was the common clinical entity $(88 \%, n=28)$. UTI was the common group of disease. Among the patients with UTI a total of 191 $(66 \%)$ patients presented with fever, 75 (26.8\%) with urinary symptoms and $35(12.2 \%)$ with abdominal tenderness. The urine culture was positive with single organism in $117(40.4 \%)$ of the patients and 99 (85\%) was Escherichia coli followed by other Enterobacteriaceae (12\%). Mixed growths were seen in $22.5 \% \quad(n=65)$. Ultrasound Scan (USS) was performed in 246 $(90 \%)$ and $128(52 \%)$ had renal parenchymal involvement and renal tract anomaly in $6(2.4 \%)$. Children under one year with proven UTI were 89 $(47 \%)$ and all of them had USS of abdomen and in these patients DMSA was performed in $42(47 \%)$, MCUG 11 (6\%) and DTPA was done in 16 (18\%). There was no significant association with maternal education and family income
There were $91(17.6 \%)$ children in the category of nephrotic syndrome with the mean age of $3.11 \pm$ 1.05 years. Renal biopsy was performed in 21 (23\%) which showed minimal change nephrotic syndrome (MCNS) in $14(67 \%)$, focal segmental glomerulosclerosis (FSGS) in 3 (14\%) and membrano-proliferative glomerulonephritis (MPGN) in 2 (9.5\%). A total of 86 (94.6\%) patients were steroid responsive. Rest of the children needed a second line immune-modulating drug to achieve remission. Recurrent admissions were seen commonly in this group of patients which accounted for $24 \%(n=22)$ who were admitted more than once. There were no cases with congenital nephrotic syndrome.

The diagnosis of nephritis in the study population was made based on the clinical features. Majority of the children presented with haematuria $(96 \%$, $n=69)$, facial puffiness $(89 \%, \quad n=64)$ and hypertension $(86 \%, \mathrm{n}=62)$. Anti streptolysin O titre (ASOT) was checked in $28(38.8 \%)$ and it was above $400 \mathrm{IU}$ in $20(71 \%)$. CKD excluding the 
renal tract anomalies were $20(3.87 \%)$ and the cause was not known in $12(60 \%)$. Three patients had CKD following haemolytic uraemic syndrome (HUS), three following glomerulonephritis and two following systemic lupus erythematosus (SLE). Eight patients were on regular dialysis and $3(30 \%)$ succumbed to the illness. Renal tract anomalies were seen in $10(1.9 \%)$ and the mean age of presentation was $8.2 \pm 1.8$ months. Antenatal scans detected the anomaly in 4 patients (40\%). The common anomaly was posterior urethral valve $(40 \%, \quad n=4)$ followed by dysplastic kidney $(30 \% \mathrm{n}=3)$ and pelvi-ureteric junction obstruction $(30 \%, \quad n=3)$. Tubulopathy and idiopathic hypercalciuria were seen in $2.1 \%$ and $2.5 \%$ respectively. Growth faltering was the commonest $(73 \%, \mathrm{n}=8)$ clinical presentation for tubulopathy and haematuria $(75 \%, n=3)$ for hypercalciuria. Isolated haematuria was the reason for referral in $4(0.77 \%)$ patients, where the diagnosis was not made due to limited facilities. There were 4 cases $(0.8 \%)$ diagnosed with Wilms tumour during the study period. They were transferred to cancer institute Maharagama for further evaluation and management.

The overall outcome of the renal diseases indicated that majority $(49.7 \%)$ were on regular follow-up and $6.1 \%$ were referred to specialised renal units. Table 4 shows the overall outcome of the renal diseases. The mortality of these patients was $0.7 \%$ of the total study population. The main reason was CKD and renal tract malignancy.

Table 4: Outcome of the renal diseases

\begin{tabular}{|l|c|}
\hline \multicolumn{1}{|c|}{ Outcome } & Number (\%) \\
\hline Discharged without any follow-up & $216(41.7)$ \\
\hline Regular follow-up & $257(49.7)$ \\
\hline Transferred to specialized renal units & $32(06.2)$ \\
\hline Haemodialysis & $08(01.5)$ \\
\hline Dead & $04(0.7)$ \\
\hline Total & $517(100.0)$ \\
\hline
\end{tabular}

\section{Discussion}

There are many reports and articles related to renal diseases in children and reports from Sri Lanka are limited to specific diseases such as CKDu, nephrotic syndrome and $\mathrm{UTI}^{3,6,7}$. The experience from a tertiary centre of the inpatient profile of renal diseases is been reported here. The neonatal and the outpatient data have not been included in the study. The reports from different parts of the world suggest that paediatric renal diseases comprise 3-8.9 \% of all patients admitted to a general pediatric ward $^{11,12}$. In our study, kidney disease accounted for $3.9 \%$ of all admissions. It also follows the other studies reflecting renal diseases as a significant cause of morbidity indicating that there may be similar genetic and environmental factors involved.

The most common age group affected in our study was children between $1-5$ years of age $(49.1 \%)$. The number of new cases each year also has been remaining relatively constant from 2015 to 2018 . Studies reported in the literature indicate a rising trend $^{11,12}$. The rising trend is not demonstrated in our study due to smaller case numbers and the possibility of not having proper screening measures to identify renal diseases. Males $(52.6 \%)$ were affected more than the females $(47.4 \%)$. Similar results were reported by various authors whereas some studies reported equal gender distributions ${ }^{13,14}$. Possible explanation may be due to male hormonal milieu having a negative impact on the kidney haemodynamics ${ }^{15}$.
UTI was the commonest cause of admission accounting for about $55.9 \%$ of all renal diagnoses. Nephrotic syndrome, another common paediatric renal problem reported in the literature, is the second most common renal diagnosis in our study accounting for $17.6 \%$. Nephrotic syndrome accounted for most multiple admissions in our study. Third common cause was acute glomerular nephritis accounting for $13.9 \%$ of renal diseases. These are potentially preventable causes of chronic kidney disease and may contribute to the CKD load of adolescents and adults in the region. Studies done in North India and Iran suggest acute glomerulonephritis and nephrotic syndrome as the common renal diseases ${ }^{13,14}$. The difference in the spectrum of diseases may be explained by the geographical variations ${ }^{14,16}$.

Even though UTI is diagnosed based on the urine culture, this study indicated children treated based on clinical grounds. Considering the clinical presentation of UTIs, unexplained fever was the commonest $(66 \%)$ followed by typical urinary symptoms $(12.2 \%)$. This is compatible with the literature available $e^{13,14}$. Considering the investigations, culture positive UTI with single organism was seen in $40.4 \%$ and the common organism was Escherichia coli. It is compatible with a study done in tertiary care centre in Sri Lanka which also showed that coliforms were isolated in $60 \%$ of the patients ${ }^{6}$. A fair number $(37 \%)$ of children with UTI had negative urine 
cultures. The literature states culture positivity is seen only $60 \%$ of the time ${ }^{14}$. The low yield may be explained by prior use of antibiotics in the community which may be the cause for the low yield in our study as well. Urine culture with mixed growth patterns was seen in $22.5 \%$ of the children indicating contamination mainly due to improper collection methods. None of the other studies demonstrated this feature; hence education regarding use of antibiotics and collection techniques must be reinforced to both medical personnel and patients ${ }^{14,16}$. Almost $90 \%$ of the children with UTI had an USS of the renal system and $52 \%$ of them had some abnormality. A clear consensus must be devised to help in selecting the patients for an USS.

The onset of disease of nephrotic syndrome was less than 5 years in our study and it was compatible with studies done in India ${ }^{17}$. ISKDC found that almost $60 \%$ of the children with minimal change nephrotic syndrome were from two to less than six years of age ${ }^{16,17}$. Majority of the children with nephrotic syndrome were steroid sensitive in our study and it was the same in other studies as well ${ }^{6}$, 16. It may be the fact that nephrotic syndrome behaves the same throughout the world even though environment, heavy metals and certain viruses play a role in this ${ }^{18}$. Glomerulonephritis was the $3^{\text {rd }}$ common condition in our study. Post infectious glomerulonephritis is the common cause of nephritis reported in literature ${ }^{19,20}$. We could not establish this fact due to limitation of resources to check the antibody titres for infections. Six patients had acute kidney injury (AKI) and the underlying causes were sepsis, snake envenomation and dehydration due to diarrhoea. Sepsis was the leading cause of AKI in other studies ${ }^{21}$. The aetiology for AKI cannot be arrived at from this study due to the low numbers. AKI due to snake envenomation has been reported in studies from India $^{19,20}$.

Positive family history of renal disease was seen in $6.2 \%$ of the study population and $88 \%$ had renal calculi. This was not related to the diseases the children had but it may be explained by the increase incidence of renal calculi and CKDu over the recent past in this part of the country ${ }^{22,23}$. The overall outcome of the renal diseases indicated that the majority $(49.9 \%)$ were on regular follow-up and $6.1 \%$ were referred to specialised renal units. Four $(0.7 \%)$ died due to renal diseases. Regular follow-up of these patients is mainly advocated to prevent further renal diseases in the patients and hence it becomes a burden to the healthcare facilities ${ }^{20}$. It can be prevented if clear protocols are available to facilitate the care of these children. Protocols should be developed to screen children at outpatient, general practitioner levels and clear guidelines on follow-up of these patients utilising the existing healthcare system in the community.

As this was a single-centre study, the extracted data may not have been an adequate representation of the entire population. Some of the aetiologies had low numbers as it was a single general paediatric unit. A prospective study is recommended to evaluate the aetiology in detail. Electronic patient management systems are useful and saves time specially to conduct retrospective studies.

This study gives an insight into the overall disease spectrum of the renal diseases in a tertiary care general paediatric unit. Infection of the renal tract was the common cause of admission in our study. Proper collection of urine and rational use of antibiotics will help in the management of UTI. Early recognition by proper investigation, treatment and regular follow-up are essential in the management of children with kidney diseases.

\section{Conclusions}

Renal diseases accounted for $3.9 \%$ of total admissions during the study period. UTI was the commonest disease $(55.9 \%)$ followed by nephrotic syndrome $(17.6 \%)$ and acute glomerulonephritis $(13.9 \%)$.

\section{References}

1. Rajapakse S, Shivanthan MC. Selvarajah M. Chronic kidney disease of unknown aetiology in Sri Lanka, International Journal of Occupational and Environmental Health 2016; 22(3): 25964.

https://doi.org/10.1080/10773525.2016.12 03097

PMid: 27399161 PMCid: PMC5102238

2. Kafle K, Balasubramanya S, Horbulyk T. Prevalence of chronic kidney disease in Sri Lanka: A profile of affected districts reliant on groundwater. Science of The Total Environment 2019; 694: 133767. https://doi.org/10.1016/j.scitotenv.2019.13 3767

PMid: 31756806

3. Agampodi SB, Amarasinghe GS, Naotunna PGCR, Jayasumana CS, Siribaddana SH. Early renal damage among children living in the region of highest burden of chronic kidney disease of unknown etiology (CKDu) in Sri Lanka. BMC Nephrology 2018; 19: Article No. 115. 
https://doi.org/10.1186/s12882-018-09118

PMid: 29769043 PMCid: PMC5956963

4. Maheswari K, Manikandasamy V, Shanmuga Arumugasamy S, Kiran, Lakshman, Brinda. Magnitude and clinico-etiological profile of renal disorders in children - a retrospective study in tertiary care hospital. Pediatric Review: International Journal of Pediatric Research 2017; 4(8): 519-24.

https://doi.org/10.17511/ijpr.2017.i08.04

5. Anders H-J. 2018 update in basic kidney research: fibrosis, inflammation, glomerular filtration and kidney disease progression. Nephrology Dialysis Transplantation 2019; 34(5): 719-23. https://doi.org/10.1093/ndt/gfz006 PMid: 30778545

6. Hathagoda K, Gunathilaka S, Dissanayake B, Tharaka I, Gunathilaka K, Jayawardane $\mathrm{R}$, et al. Bacterial pathogens causing urinary tract infections in children and their antimicrobial susceptibility patterns in a tertiary care hospital in Sri Lanka. Sri Lankan Journal of Infectious Diseases 2018; 8(2): 93-9.

https://doi.org/10.4038/sljid.v8i2.8222

7. Thalgahagoda S, Abeyagunawardena S, Karunadasa U, Abeyagunawardena A. Managing a child with nephrotic syndrome. Sri Lanka Journal of Child Health 2017; 46(3):267-72.

https://doi.org/10.4038/sljch.v46i3.8330

8. National Kidney Foundation. Kidney disease outcome quality initiative clinical guidelines for chronic disease: Evaluation, classification and stratification, Part IV. Definition and classification of stages of chronic kidney disease. American Journal of Kidney Disease 2002; 39:S46-75 https://doi.org/10.1053/ajkd.2002.30943

9. The Kidney Disease: Improving Global Outcomes (KDIGO) 2012 Clinical Practice Guideline for the Evaluation and Management of Chronic Kidney Disease (CKD) https://kdigo.org/guidelines/ckdevaluation-and-management/. Accessed $1^{\text {st }}$ May 2020.

10. A report for the International Study of Kidney Disease in Children. https://www.kidney-
international.org/article/S00852538(15)32626-0/pdf. Accessed $1^{\text {st }}$ May 2020

11. Sangla L, Ksoo R. Rapthap K. Pattern of paediatric kidney diseases in a tertiary care centre in Northeast India: a 5 year retrospective analysis. Journal of Paediatric Nephrology 2018; 6(2): 6-10.

12. Yadav SP, Shah GS, Mishra OP, Baral N. Pattern of renal diseases in children: A developing country experience. Saudi Journal of Kidney Diseases and Transplantation 2016; 27(2): 371-6. https://doi.org/10.4103/1319-2442.178565 PMid: 26997393

13. Sonowal R. Profile of renal diseases in North-East Indian children. Saudi Journal of Kidney Diseases and Transplantation 2019; 30(5): 1151-5.

https://doi.org/10.4103/1319-2442.270272 PMid: 31696855

14. Bhatta NK, Shrestha P, Budhathoki S, Kalakheti B. Profile of renal diseases in Nepalese children. Kathmandu University Medical Journal 2008; 6(2):191-4.

15. Neugarten J, Golastaneh L. Gender and the prevalence and progression of renal disease Advances in Chronic Kidney Disease 2013; 20(5): 390-5.

https://doi.org/10.1053/j.ackd.2013.05.004 PMid: 23978543

16. Dufek S, Cheshire C, Levine AP, Trompeter RS, Issler N, Stubbs M, et al. Genetic identification of two novel loci associated with steroid-sensitive nephrotic syndrome. Journal of the American Society of Nephrology 2019; 30(8): 137584.

https://doi.org/10.1681/ASN.2018101054 PMid: 31263063

17. Nephrotic syndrome in children: Prediction of histopathology from clinical and laboratory characteristics at time of diagnosis. A report of the international study of kidney disease in children. Kidney International 1978; 13:159-65. https://doi.org/10.1038/ki.1978.23 PMid: 713276

18. Eddy AA, Symons JM. Nephrotic syndrome in childhood. Lancet 2003; 362(9384): 629-39. 
https://doi.org/10.1016/S01406736(03)14 184-0

19. Derakhshan A, Al Hashemi GH, Fallahzadeh MH. Spectrum of in-patient renal diseases in children: A report from Southern part Islamic Republic of Iran. Saudi Journal of Kidney Diseases and Transplantation 2004; 15:12-7.

20. Gunasekaran K, Krishnamurthy S, Mahadevan S, Harish BN, Kumar AP. Clinical characteristics and outcome of post-infectious glomerulonephritis in children in Southern India: A prospective study. Indian Journal of Pediatrics 2015; 82(10: 896-903.

https://doi.org/10.1007/s12098-015-17520

PMid: 25893528

21. Krishnamurthy S, Narayanan P, Prabha S, Mondal N, Mahadevan S, Biswal N, et al. Clinical profile of acute kidney injury in a pediatric intensive care unit from Southern India: A prospective observational study. Indian Journal of Critical Care Medicine 2013; 17(4):207-13. https://doi.org/10.4103/0972-5229.118412 PMid: 24133327 PMCid: PMC3796898

22. Abeygunasekera AM. Urinary stone disease in Sri Lanka. Ceylon Medical Journal 2004; 49: 41-3.

https://doi.org/10.4038/cmj.v49i2.3258

PMid: 15334796

23. Saravanapavanathan T, Magesweran R. Nature and composition of urinary calculi: A preliminary study in northern Sri Lanka. Jaffna Medical Journal 1988; 23: 37-42. 